\title{
Tick-transmitted human infections in Asia
}
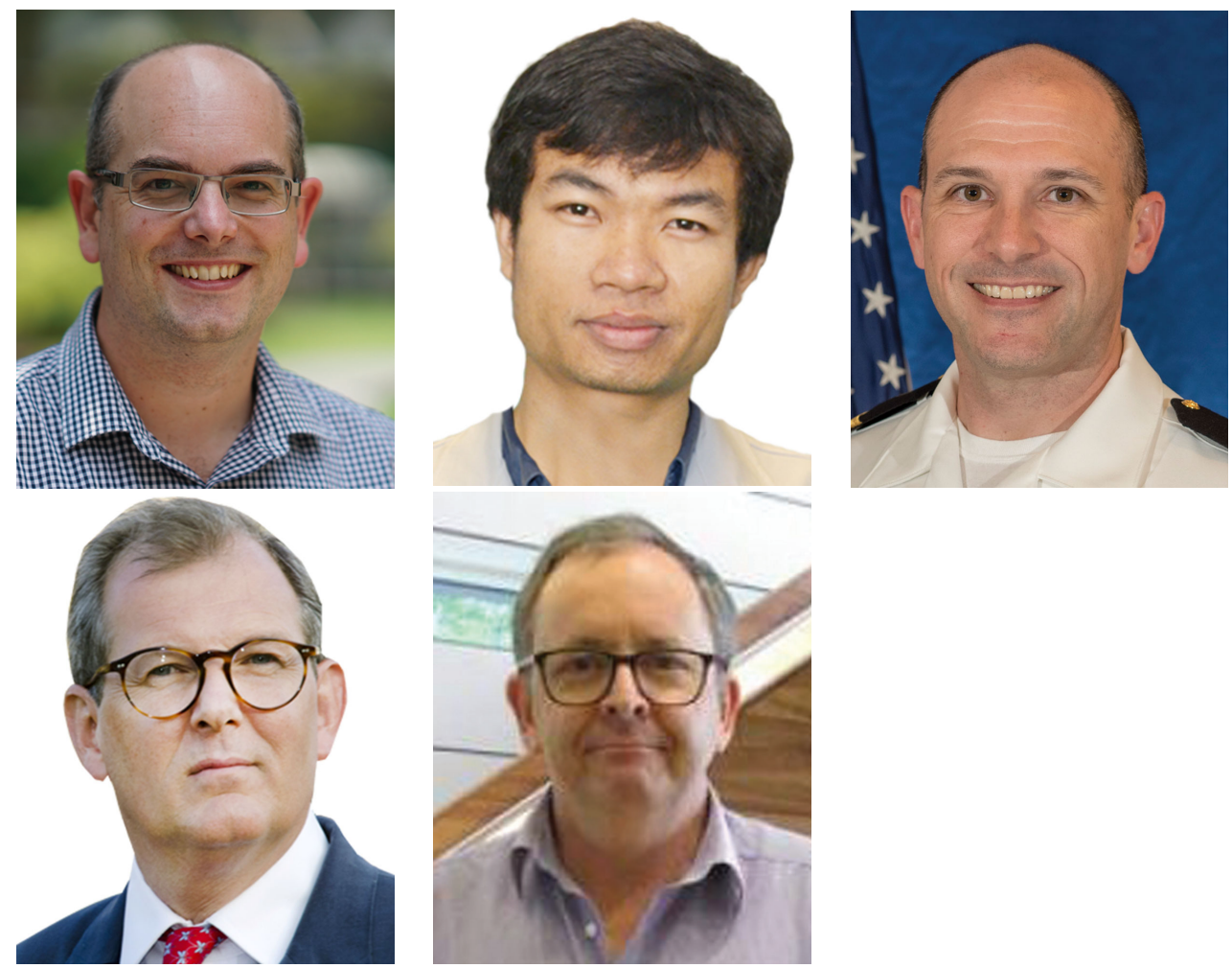

Matthew T Robinson ${ }^{\mathrm{A}, \mathrm{B}, \mathrm{E}}$, Khamsing Vongphaylotb ${ }^{\mathrm{C}}$, Jeffrey $\mathrm{CHertz}^{\mathrm{D}}$, Paul Brey ${ }^{\mathrm{C}}$ and Paul N Newton $^{\mathrm{A}, \mathrm{B}}$

${ }^{A}$ Lao-Oxford-Mahosot Hospital-Wellcome Trust Research Unit (LOMWRU), Microbiology Laboratory, Mahosot Hospital, Vientiane, Lao PDR

${ }^{\mathrm{B}}$ Centre for Tropical Medicine and Global Health, Nuffield Department of Clinical Medicine, University of Oxford, Oxford, United Kingdom

${ }^{\mathrm{C}}$ Institut Pasteur du Laos, Vientiane, Lao PDR

${ }^{D}$ U.S. Naval Medical Research Unit TWO, Sembawang, Singapore

${ }^{\mathrm{E}}$ Tel: +856 (0) 21 250752, Email: matthew.r@tropmedres.ac

Vector-borne pathogens of human significance cause a predicted $17 \%$ of infectious diseases worldwide, of which, $\sim 23 \%$ are tick transmitted ${ }^{1}$. Although second to mosquitoes in terms of impact, ticks are thought to carry a greater diversity of pathogens than other arthropod vectors $^{2}$. Asia is a key region for tick-borne pathogens, with tick species typically restricted to latitudes below $60-55^{\circ} \mathrm{N}^{3}$ where the climate is warmer and wetter - from the steppe regions of Russia to the tropical rainforests of South East Asia.

There are approximately 896 species of tick (Ixodidae, Argasidae and Nuttalliellidae) worldwide ${ }^{4}$. In Asia the knowledge of key species is still limited, especially in the Southeast. Tick species that may transmit specific pathogens are highly dependent on distribution, with studies described below primarily identifying
Ixodes spp, Haemaphysalis spp., Hyalomma spp. and Dermacentor spp. as important vectors for various pathogens.

Despite the prevalence of ticks and the clinical importance of the pathogens transmitted, very little information is available on the disease burden and distribution of tick-transmitted infections in Asia, particularly outside of Russia, China, Japan and Korea. This is most likely due to lack of research in ticks and tick-borne diseases (TBD) outside of the more developed northern Asian countries, and a lack of knowledge in the healthcare systems of LMICs (lower to middle income countries) as many TBD infections have similar clinical presentations and available diagnostics may be limited. Knowledge of TBDs is highly dependent on whether the diseases are notifiable within the country; in Russia for instance, seven TBDs are reportable providing incidence data, but little is known about other non-reportable infections ${ }^{5}$. 
Table 1. Tick-borne rickettsias identified in Asia. Identification either by serology (S), PCR (G) or isolation (I) from patients and/or ticks within Asia.

\begin{tabular}{|c|c|c|c|c|c|}
\hline \multicolumn{3}{|c|}{ Known pathogenic rickettsias } & \multicolumn{3}{|c|}{ Rickettsias of unknown pathogenicity } \\
\hline Species & $\begin{array}{l}\text { Human } \\
\text { ID? }\end{array}$ & Tick ID? & Species & $\begin{array}{l}\text { Human } \\
\text { ID? }\end{array}$ & $\begin{array}{l}\text { Tick } \\
\text { ID? }\end{array}$ \\
\hline R. aeschlimannii & - & G & R. asiatica & - & I \\
\hline R. conorii indica & $\mathrm{G} / \mathrm{S}$ & I & R. bellii & - & G \\
\hline R. heilongjiangensis & G & $\mathrm{G} / \mathrm{l}$ & R. hoogstraalii & - & G \\
\hline R. helvetica & S & - & R. tarasevichiae & - & G \\
\hline R. honei & $\mathrm{G} / \mathrm{S}$ & G & Candidatus R. gannanii & - & G \\
\hline R. japonica & G/l & $\mathrm{G} / \mathrm{l}$ & Candidatus R. khammouanensis & - & G \\
\hline R. massiliae & G & G & Candidatus $\mathrm{R}$ laoensis & - & G \\
\hline R. monacensis & - & G & Candidatus R. mahosotii & - & G \\
\hline R. raoultii & G & $\mathrm{G} / \mathrm{l}$ & Candidatus R. principis & - & G \\
\hline R. rickettsii & S & - & Candidatus R. tibetani & - & G \\
\hline R. sibirica sibirica & G & $\mathrm{G} / \mathrm{l}$ & & & \\
\hline R. sibirica mongolitimonae & G & G & & & \\
\hline R. slovaca & - & $G$ & & & \\
\hline R. tamurae & $\mathrm{G} / \mathrm{S}$ & $\mathrm{G} / \mathrm{I}$ & & & \\
\hline Candidatus R. kellyi & $\mathrm{G}$ & - & & & \\
\hline
\end{tabular}

In Russia approximately 0.5 million tick bites are reported each year, with an estimated $\leq 2 \%$ resulting in clinical infections, although this is likely to be much higher, particularly in rural regions $^{5}$. In Japan, 12 TBDs are reportable, while in Korea, six diseases of potential tick origin are reportable $e^{6,7}$. TBD in Asia can be categorised into four distinct groups: rickettsias, other bacterial pathogens, protozoa and viruses.

\section{Rickettsias}

The rickettsias form the largest group of TBD in Asia. Although globally distributed, at least thirteen clinically important rickettsial species have been identified throughout Asia (east of the Caspian Sea) in either patients or ticks ${ }^{8-11}$. Currently a further 10 (including candidatus species) have been identified in ticks although their implications for public health is uncertain ${ }^{8,12-14}$ (Table 1). Often, identification in patients is made by serological techniques, limiting identification to non-specific genus-level rather than species-level, which may obscure the clinically important species circulating in the region. Symptoms for infections are variable, with most causing fever, chills, headache, malaise and myalgia with a variable proportion developing a maculopapular rash. $R$. sibirica results in a lymphangitis-associated rickettsiosis ${ }^{15}$.

\section{Other bacterial pathogens}

Closely related to the rickettsias are Anaplasma and Ehrlichia. A. phagocytophilum is the agent of Human Granulocytic Anaplasmosis (HGA) ${ }^{16}$, while E. chaffeensis is the cause of Human Monocytic Ehrlichiosis (HME) ${ }^{16,17}$. Both share similar symptoms including fever, headache, leukocytopenia, with neurological symptoms more common in $\mathrm{HME}^{18}$. Borreliosis is becoming more important throughout the region, with Borrelia afzelii and Bo. garinii being the main species in Asia, although a Bo. valaisianarelated sp. has also been identified in patients ${ }^{12,19,20}$. Despite its dominance in the western hemisphere, Bo. burgdorferi sensu stricto has only been isolated from rodents in Asia ${ }^{19,20}$. Borreliosis may present with erythema migrans, fever, headaches and fatigue, and in a minority, cardiac and central and peripheral central nervous system abnormalities ${ }^{19}$. Although the following human pathogens (Francisella spp., Bartonella spp., Brucella spp. and Coxiella spp.) have been identified in ticks in Asia, the tick-human 
route of transmission for these four organisms is highly disputed or considered infrequent. Infection is more likely through other routes such as other vectors, direct contact with animals, food items or aerosols; nevertheless, ticks may still play a vital, yet indirect role in disease incidence. Francisella tularensis has been detected in ticks from Japan, China and Thailand ${ }^{21,22}$, while $F$. novicida, has been isolated from a patient in Thailand ${ }^{23}$. At least 15 species of Bartonella are known in Asia, some of which have been identified in ticks ${ }^{24}$. There are reports of clinical Bartonella spp. infections in China, Thailand, Japan and Korea ${ }^{24,25}$, although these may be due to transmission via fleas or mammalian contact. The greatest human incidences of brucellosis infections are reported from central Asia ${ }^{26}$. Brucella melitensis and Br. abortus (the most pathogenic species) have been identified in ticks and shown to be transmitted ${ }^{27}$. A number of tick species have been shown to harbour Coxiella burnetii (the etiologic agent of $\mathrm{Q}$ fever) in Malaysia, Laos and Thailand ${ }^{12,22,28}$. Transmission from ticks to mammalian hosts has been shown to occur experimentally but it remains to be seen if this is a viable route for human infections.

\section{Protozoa}

Although predominantly recognised as a TBD of veterinary importance, cases of human babesiosis have been identified throughout China (including the China-Myanmar boarder), Russia, Japan and Korea $^{29}$. Infections are predominantly Babesia microti, although $B a$. divergens and $B a$. venatorum have also been identified. Clinical symptoms are similar to malaria infections and therefore often result in misdiagnosis and under-reporting of this pathogen. Ixodes persulcatus is considered the key tick species for transmission $^{29}$.

\section{Viruses}

Tick-borne encephalitis viruses (TBEV) have been identified in both ticks and patients across $\mathrm{Asia}^{30}$, including serological evidence in rodents and humans in Vietnam ${ }^{31}$. Infection may result in central nervous system abnormalities. Of the Bunyavirales, outbreaks of Crimean-Congo haemorrhagic fever (CCHF) have been reported in China, the first of which was in Xinjiang Province in $1965^{32}$ and in Pakistan and India ${ }^{33}$. Clinical symptoms include severe fever, haemorrhage, fatigue, myalgia, oliguria and disturbance of consciousness. Severe Fever with Thrombocytopenia Virus (SFTV) has been reported in China, Japan and South Korea and is transmitted by Haemaphysalis longicornis ticks. SFTV is characterised by fever, thrombocytopenia, leukocytopenia, increased serum liver enzyme levels, and organ failure ${ }^{34}$. Powassan virus is a rare, yet potentially fatal neurotropic virus seemingly restricted in Asia to Far Eastern Russia region. Symptoms vary between patients, making diagnosis difficult, but may rapidly develop into more severe symptoms including neurological defects $^{35}$. Kyasanur Forest Virus (KFV, a flavivirus) is found in southern India, presenting with haemorrhagic and neurological symptoms and is thought to be transmitted predominantly by Haemaphysalis spinigera ${ }^{36}$.

The zoonotic nature of TBDs, combined with a higher proportion of rural populations in Asia, heightens the risk of exposure to TBDs and places a significant weight on scarce public health resources. Surveillance of ticks for potential human pathogens across Asia is needed to alert for clinical problems ${ }^{12}$. Improved diagnostics, evidence for appropriate management and public and policy engagement are very much in need, supported by validated survey and surveillance research to better understand the distribution and epidemiology of these potentially life-threatening diseases.

\section{Disclaimers}

JCH is a military service member or federal/contracted employee of the United States government. The views expressed in this article reflect the results of research conducted by the author and do not necessarily reflect the official policy or position of the Department of the Navy, Department of Defence, nor the United States Government.

\section{Conflicts of interest}

The authors declare no conflicts of interest.

\section{Acknowledgements}

MR, PN and LOMWRU are funded by the Wellcome Trust of Great Britain (Grant number 089275/H/09/Z)

\section{References}

1. WHO (2017) Vector-borne diseases. http://www.who.int/news-room/fact-sheets/ detail/vector-borne-diseases

2. Ahmed, J. et al. (2007) Current status of ticks in Asia. Parasitol. Res. 101(Suppl 2), S159-S162. doi:10.1007/s00436-007-0696-3

3. Stanek, G. et al. (2012) Lyme borreliosis. Lancet 379, 461-473. doi:10.1016/ S0140-6736(11)60103-7

4. Guglielmone, A.A. et al. (2010) The Argasidae, Ixodidae and Nuttalliellidae (Acari: Ixodida) of the world: a list of valid species names. Zootaxa 2528, 1-28.

5. Dedkov, V.G. et al. (2017) The burden of tick-borne diseases in the Altai region of Russia. Ticks Tick Borne Dis. 8, 787-794. doi:10.1016/j.ttbdis.2017.06.004

6. Yamaji, K. et al. (2018) Distribution of tick-borne diseases in Japan: Past patterns and implications for the future. J. Infect. Chemother. 24, 499-504. doi:10.1016/j.jiac.2018.03.012

7. Park, S. and Cho, E. (2014) National Infectious Diseases Surveillance data of South Korea. Epidemiol. Health 36, e2014030. doi:10.4178/epih/e2014030 
8. Parola, P. et al. (2013) Update on tick-borne rickettsioses around the world: a geographic approach. Clin. Microbiol. Rev. 26, 657-702. doi:10.1128/CMR. 00032-13

9. Imaoka, K. et al. (2011) The first human case of Rickettsia tamurae infection in Japan. Case Rep. Dermatol. 3, 68-73. doi:10.1159/000326941

10. Wei, Q.Q. et al. (2015) The first detection of Rickettsia aeschlimannii and Rickettsia massiliae in Rhipicephalus turanicus ticks, in northwest China. Parasit. Vectors 8, 631. doi:10.1186/s13071-015-1242-2

11. Lai, C.H. et al. (2014) Human spotted fever group rickettsioses are underappreciated in southern Taiwan, particularly for the species closely-related to Rickettsia felis. PLoS One 9, e95810. doi:10.1371/journal.pone.0095810

12. Taylor, A.J. et al. (2016) Large-scale survey for tickborne bacteria, Khammouan Province, Laos. Emerg. Infect. Dis. 22, 1635-1639. doi:10.3201/eid2209.151969

13. Guo, L.P. et al. (2016) Emerging spotted fever group rickettsiae in ticks, northwestern China. Ticks Tick Borne Dis. 7, 1146-1150. doi:10.1016/j.ttbdis.2016. 08.006

14. Han, R. et al. (2018) Molecular prevalence of spotted fever group rickettsiae in ticks from Qinghai Province, northwestern China. Infect. Genet. Evol. 57, 1-7. doi:10.1016/j.meegid.2017.10.025

15. Kuscu, F. et al. (2017) Rickettsia sibirica mongolitimonae infection, Turkey, 2016. Emerg. Infect. Dis. 23, 1214-1216. doi:10.3201/eid2307.170188

16. Zhang, L. et al. (2014) Rural residents in China are at increased risk of exposure to tick-borne pathogens Anaplasma phagocytophilum and Ehrlichia chaffeensis. BioMed Res. Int. 2014, 313867.

17. Heppner, D.G. et al. (1997) Human ehrlichiosis in Thailand. Lancet 350 785-786. doi:10.1016/S0140-6736(05)62571-8

18. Ismail, N. et al. (2010) Human ehrlichiosis and anaplasmosis. Clin. Lab. Med. 30, 261-292. doi:10.1016/j.cll.2009.10.004

19. Ni, X.B. et al. (2014) Lyme borreliosis caused by diverse genospecies of Borrelia burgdorferi sensu lato in northeastern China. Clin. Microbiol. Infect. 20, 808-814. doi:10.1111/1469-0691.12532

20. Hao, Q. et al. (2011) Distribution of Borrelia burgdorferi sensu lato in China. J. Clin. Microbiol. 49, 647-650. doi:10.1128/JCM.00725-10

21. Suzuki, J. et al. (2016) Detection of Francisella tularensis and analysis of bacterial growth in ticks in Japan. Lett. Appl. Microbiol. 63, 240-246. doi:10.1111/lam.12616

22. Sumrandee, C. et al. (2016) Molecular detection of Rickettsia, Anaplasma, Coxiella and Francisella bacteria in ticks collected from Artiodactyla in Thailand. Ticks Tick Borne Dis. 7, 678-689. doi:10.1016/j.ttbdis.2016.02.015

23. Leelaporn, A. et al. (2008) Francisella novicida bacteremia, Thailand. Emerg. Infect. Dis. 14, 1935-1937. doi:10.3201/eid1412.080435

24. Saisongkorh, W. et al. (2009) Emerging Bartonella in humans and animals in Asia and Australia. J. Med. Assoc. Thai. 92, 707-731.

25. Kosoy, M. et al. (2010) Identification of Bartonella infections in febrile human patients from Thailand and their potential animal reservoirs. Am. J. Trop. Med. Hyg. 82, 1140-1145. doi:10.4269/ajtmh.2010.09-0778

26. Rubach, M.P. et al. (2013) Brucellosis in low-income and middle-income countries. Curr. Opin. Infect. Dis. 26, 404-412. doi:10.1097/QCO.0b013e3283 638104

27. Wang, Q. et al. (2018) Brucella melitensis and B. abortus in eggs, larvae and engorged females of Dermacentor marginatus. Ticks Tick Borne Dis. 9, 1045-1048. doi:10.1016/j.ttbdis.2018.03.021

28. Khoo, J.J. et al. (2016) Coxiella detection in ticks from wildlife and livestock in Malaysia. Vector Borne Zoonotic Dis. 16, 744-751. doi:10.1089/vbz.2016.1959

29. Zhou, X. et al. (2014) Human babesiosis, an emerging tick-borne disease in the People's Republic of China. Parasit. Vectors 7, 509.

30. Süss, J. (2011) Tick-borne encephalitis 2010: epidemiology, risk areas, and virus strains in Europe and Asia-an overview. Ticks Tick Borne Dis. 2, 2-15. doi:10.1016/j.ttbdis.2010.10.007

31. Van Cuong, N. et al. (2015) Rodents and risk in the Mekong Delta of Vietnam: seroprevalence of selected zoonotic viruses in rodents and humans. Vector Borne Zoonotic Dis. 15, 65-72. doi:10.1089/vbz.2014.1603
32. Zhang, Y. et al. (2018) Isolation, characterization, and phylogenetic analysis of two new Crimean-Congo hemorrhagic fever virus strains from the northern region of Xinjiang Province, China. Virol. Sin. 33, 74-86. doi:10.1007/s12250018-0020-7

33. Lahariya, C. et al. (2012) Emergence of viral hemorrhagic fevers: is recent outbreak of Crimean Congo Hemorrhagic Fever in India an indication? J. Postgrad. Med. 58, 39-46. doi:10.4103/0022-3859.93251

34. Park, S.W. et al. (2014) Severe fever with thrombocytopenia syndrome virus, South Korea, 2013. Emerg. Infect. Dis. 20, 1880-1882. doi:10.3201/ eid2011.140888

35. Fatmi, S.S. et al. (2017) Powassan virus-a new reemerging tick-borne disease Front. Public Health 5, 342. doi:10.3389/fpubh.2017.00342

36. Holbrook, M.R. (2012) Kyasanur forest disease. Antiviral Res. 96, 353-362. doi:10.1016/j.antiviral.2012.10.005

\section{Biographies}

Dr Matthew Robinson is Head of Molecular Bacteriology at the Lao-Oxford-Mahosot Hospital-Wellcome Trust Research Unit (LOMWRU) based in Vientiane, Lao PDR, and is part of the MORU Tropical Network. The Molecular Bacteriology group supports the microbiology laboratory with molecular diagnostics, as well as carrying out research on the causes of febrile illnesses in Laos and SE Asia, and evaluating novel diagnostic assays for potential use in low resource settings.

Dr Khamsing Vongphayloth is a medical entomologist based at Institut Pasteur of Laos in Vientiane, Lao PDR. His research covers the systematics of arthropod vectors (mosquitoes, ticks, chigger mites and sandflies) and pathogens related to arthropods, in particular bacteria and arboviruses. He is currently working on the biology, ecology and taxonomy of arthropod vectors (mosquitoes, ticks and sandflies) in Laos, and the molecular techniques for identification of these arthropods.

Lieutenant Commander Jeffrey Hertz is the Head of the Laboratory and Field Research Department at U.S. Naval Medical Research Unit TWO, headquartered in Singapore. In this role, he oversees numerous bio-surveillance projects at the NAMRU-2 main laboratory in Phnom Penh, Cambodia and at dozens of governmental and non-governmental collaborative laboratories spanning seven countries. Dr Hertz received his Master's and Doctoral medical entomology training at the University of Florida in the United States

Dr Paul Brey was appointed Director General of Institut Pasteur of Laos in Vientiane, Laos, a Lao national institute, a project that he lead from its inception in 2004 to its completion in 2012. The Minister of Health of the Lao People's Democratic Republic has since given Dr Brey the task to direct and develop Institut Pasteur of Laos into a regional center of excellence for infectious disease research and training. In addition to his role as director, 
Dr Brey also is head of the Medical Entomology Unit at IP Laos. Paul Brey's research has focused on insect innate immunity, insect genomics, host-parasite interactions and more recently on the natural history of pathogen-arthropod transmission cycles and viral/bacterial pathogen discovery in arthropods. He is the author of 90 peer-reviewed scientific articles. He also serves or has served on several scientific advisory boards at Institut Pasteur, at the World Health Organization, and the French Ministry of Science and Technology and is presently the Co-President of the 'Fondation Pasteur Suisse' Scientific Advisory Board.

Professor Paul Newton is an infectious disease physician, based from the Centre of Tropical Medicine and Global Health at the University of Oxford, and directs the Lao-Oxford-Mahosot Hospital-Wellcome Trust Research Unit (LOMWRU) in Vientiane, Lao PDR. He works on the epidemiology, diagnosis and management of fevers, especially rickettsial infections, in Asia and on the global issue of medicine quality. He is also Head of the Medicine Quality Group of the Worldwide Antimalarial Resistance Network (WWARN) in Oxford. He is Professor of Tropical Medicine at Oxford, an Honorary Professor at the London School of Hygiene and Tropical Medicine and at the National University of Laos and Visiting Scholar at Boston University, USA. 